\title{
A adoção de metodologias ativas, software livres e repositórios gratuitos no ensino do processamento e interpretação de imagens de Radar Sentinel-1
}

Jean Carlos Dias - PPGET/IFAM - jeandias.ifam@gmail.com Andréa Pereira Mendonça - PPGET/IFAM - andrea.mendonca@ifam.edu.br

\begin{abstract}
Resumo: Neste artigo apresenta-se uma proposta de ensino-aprendizagem baseada no emprego de metodologias ativas e softwares livres, para capacitar graduandos e profissionais recémegressos de cursos superiores relacionados ao Meio Ambiente a processar e interpretar imagens de Radar Sentinel-1. Os resultados observados, a partir da realização de um estudo de caso, demonstraram evidências da aprendizagem dos alunos quanto ao uso de tecnologias, ao processamento de imagens de Radar orbital e interpretação dessas imagens para a realização de diagnóstico ambiental. Como contribuição deste trabalho, foi produzido um Guia para professores de geotecnologias de Cursos Superiores para favorecer o ensino de interpretação e análise de imagens de Radar Sentinel-1.
\end{abstract}

Palavras-chave: Ensino de Sensoriamento Remoto. Geotecnologias. Metodologias Ativas. Software livre.

\section{The adoption of active methodologies, free software and free repositories in the teaching of the Radar Sentinel-1 image processing and interpretation}

\begin{abstract}
In this paper we introduce a teaching-learning proposal based on active methodologies and free software, aimed to undergraduate students and recent graduates of higher courses of Environmental areas, that is devoted to qualify the target audience to Sentinel-1 Radar image processing and interpretation. The observed results, from the realization of a case study, showed evidence of the students learning regarding the use of technologies, the processing of orbital Radar images and the interpretation of these images for the performance of environmental diagnosis. As a contribution of this work, a Guide for Geotechnology Teachers from Higher Courses was produced to support them in the teaching of interpretation and analysis of Sentinel-1 Radar images.
\end{abstract}

Keywords: Remote Sensing Teaching. Geotechnologies. Active Methodologies. Free Software.

\section{Introdução}

Nos últimos anos, as informações obtidas por meio de dados de satélite tornaram-se cada vez mais disponíveis e populares na rotina do cidadão comum. Essas informações, obtidas por meio de um conjunto de técnicas que fazem parte de uma atividade denominada sensoriamento remoto ${ }^{1}$, estão presentes na sociedade nas mais diversas formas, como por exemplo, nas previsões meteorológicas, nos monitoramentos ambientais, no controle de tráfego aéreo e urbano, dentre muitas outras aplicações.

O ensino das técnicas de sensoriamento remoto, por meio da interpretação de dados de satélite, é uma realidade no contexto educacional brasileiro, principalmente nos cursos superiores relacionados ao Meio Ambiente (Engenharia Ambiental, Geoprocessamento, Engenharia Cartográfica, entre outros). Entende-se que esses dados, originários de satélites, possuam a capacidade de descrever as condições ambientais de

\footnotetext{
${ }^{1}$ Denomina-se Sensoriamento Remoto a obtenção de informações sobre um alvo ou evento específico, sem necessariamente entrar em contato ou estar próximo do mesmo (Novo, 2010). V. $18 \mathrm{~N}^{\mathrm{o}} 1$, julho, 2020 RENOTE DOI:
} 
uma área em estudo com precisão, fornecendo informações suficientes para avaliar as consequências de ações ocorridas durante um determinado período de tempo e projetar os impactos de ocorrências futuras, sejam elas resultantes de interações dos meios físico, biológico ou socioeconômico.

Todavia, mesmo nos cursos superiores relacionados ao Meio Ambiente, constata-se uma lacuna no ensino da interpretação de dados satelitais oriundos de sensores ativos, como as imagens de Radar, ainda que se trate de um recurso tecnicamente muito importante para a realização de análises ambientais na maior parte do território brasileiro. Os motivos dessa lacuna estão relacionados com diversos fatores, dentre eles, o alto custo para aquisição de imagens e softwares específicos para processamento dessas imagens (Dias; Mendonça, 2019).

Em que pese esse tipo de dificuldade, o cenário atual é amplamente favorável a inserção do ensino do processamento e interpretação de imagens de Radares orbitais nos projetos pedagógicos de cursos superiores relacionados ao Meio Ambiente, pois, atualmente, registra-se uma oferta crescente de sistemas de geoprocessamento e insumos gratuitos disponibilizados para aplicação em atividades profissionais $\mathrm{e}$ acadêmicas.

Sobre esse assunto, com relação às iniciativas educacionais no ensino de geotecnologias, observa-se que a adoção das metodologias ativas é uma tendência que está se tornando uma realidade no contexto educacional brasileiro. Como exemplo, pode-se citar o projeto Geotecnologias Digitais no Ensino - Universidade Federal Fluminense (GEODEN, 2019), o projeto Atlas Escolar da Região Metropolitana de Campinas (EMBRAPA, 2020) e as atividades hands-on learning propostas pela Agência Espacial Brasileira para o ensino de tecnologias espaciais nas escolas públicas (AEB, 2019).

Diante desse cenário, considerando a importância desse assunto e os desafios mencionados anteriormente, buscando contribuir para o ensino do processamento e interpretação das imagens de Radares orbitais em cursos superiores relacionados ao Meio Ambiente, mais especificamente das imagens do Radar Sentinel-1, foi elaborada uma proposta de ensino-aprendizagem, baseada no emprego de software livre, repositórios gratuitos e estruturada de acordo com algumas tendências da pesquisa em ensino, tais como, Aprendizagem Baseada em Projetos, Sala de Aula Invertida e Alinhamento Construtivo, com o intuito de proporcionar aos alunos uma aprendizagem ativa de maneira prática e significativa, solucionando problemas reais condizentes com os seus cotidianos.

Essa proposta de ensino-aprendizagem, cujos conteúdos e práticas foram planejados de modo a atender diferentes cursos superiores da área de Meio Ambiente, foi avaliada por meio de um estudo de caso, realizado no período 06 a 10 de agosto de 2018, em uma instituição federal de ensino superior, com 12 (doze) participantes. Dados os significativos resultados obtidos no estudo de caso, a proposta de ensinoaprendizagem foi sistematizada, posteriormente, em um produto educacional intitulado 'Curso de Introdução ao Processamento e à Interpretação de Imagens Sentinel-1' e submetido à apreciação de um comitê ad hoc, composto por professores que lecionam formações relacionadas com sensoriamento remoto e geotecnologias, a fim de avaliar a replicabilidade desse produto educacional por outros professores.

Os fundamentos desse estudo, os detalhes da aplicação, o percurso metodológico para avaliação da proposta de ensino-aprendizagem e do produto educacional, assim como os resultados obtidos, serão apresentados nas próximas seções. 


\section{O Sensoriamento Remoto e as Metodologias Ativas}

A fundamentação teórica desta pesquisa foi estruturada em torno de 04 (quatro) temáticas, as quais embasaram os aspectos técnicos e pedagógicos da proposta de ensino-aprendizagem, sendo elas: Sensoriamento Remoto e a sua relação com o Ensino; Aprendizagem Baseada em Projetos; Sala de Aula Invertida e Alinhamento Construtivo, essas três últimas relacionadas a metodologias ativas.

\subsection{Sensoriamento Remoto e a sua relação com o Ensino}

Pode-se entender sensoriamento remoto como sendo a atividade de observar o planeta utilizando sensores de observação muito acima do solo, sendo que esses sensores, normalmente instalados em satélites, podem registrar não somente informações na faixa do visível, mas também a radiação em outros comprimentos de onda, como por exemplo, o infravermelho e as microondas. (Souza, 2010). Do ponto de vista técnicocientífico, as imagens de sensoriamento remoto vêm servindo como fonte de dados para estudos e levantamentos geológicos, ambientais, agrícolas, cartográficos, florestais, urbanos, oceanógrafos, entre outros.

Entende-se que a seleção adequada das imagens de satélite empregadas em uma análise pode interferir significativamente nos resultados obtidos, uma vez que a baixa resolução espacial ou séries temporais incompletas podem ocasionar a insuficiência de dados e, por conseguinte, obrigar a realização de inspeções de campo para complementar as informações importantes para o estudo (Simon; Cunha, 2008). As imagens de Radar, além de possuírem excelente resolução espacial, não sofrem a interferência de nuvens e, portanto, são disponíveis o ano inteiro, inclusive a noite (Jensen, 2009).

No Brasil, a presença de nuvens, que é um aspecto limitante para a obtenção de informações na faixa do visível, não representa uma situação excepcional, pois, o regime climático brasileiro, com exceção das áreas semi-áridas do nordeste, é predominantemente úmido em todas as regiões do país, situação que favorece a presença de nuvens em grande parte do ano (IBGE, 2002).

Considerando os conteúdos didáticos, existe ainda no Brasil uma defasagem com relação às produções bibliográficas sobre sensoriamento remoto, todavia, pode-se afirmar que as literaturas especializadas, escritas ou traduzidas para o português, são cada vez mais disponíveis (Dias; Mendonça, 2019).

Sobre a viabilidade econômica do ensino das técnicas de sensoriamento remoto por meio de imagens de satélite, identifica-se que os recursos tecnológicos estão cada vez mais disponíveis e de forma gratuita. Nesse contexto, destacam-se as imagens do Radar Sentinel-1, as imagens ópticas Landsat-8, o sistema de informação geográfica QGIS e o sistema de processamento digital de imagens SNAP.

Embora haja vários fatores favoráveis ao ensino de sensoriamento remoto por meio de imagens de Radar, tanto do ponto de vista de literatura, quanto do ponto de vista tecnológico, ainda é verificado uma lacuna no currículo dos cursos superiores. Segundo Dias e Mendonça (2019), apesar do tema 'sensoriamento remoto' ser uma realidade nos cursos superiores relacionados ao Meio Ambiente, é praticamente inexistente a previsão do ensino da interpretação de imagens de Radares orbitais nos Projetos Pedagógicos desses cursos de graduação (Dias; Mendonça, 2019).

Tendo em vista esta lacuna no ensino, foi elaborada uma proposta de ensinoaprendizagem pautada em metodologias ativas, mais especificamente, na aprendizagem baseada em projetos $(\mathrm{ABP})$ que adota a resolução de problemas contextualizados com o futuro contexto profissional desses estudantes, assim como no modelo de sala de aula invertida, que mescla aprendizagem de conteúdos de maneira on-line, adquiridos de V. $18 \mathrm{~N}^{\circ} 1$, julho, 2020 RENOTE DOI: 
forma autônoma pelo aluno, deixando o tempo de sala de aula dedicado a resolução de problemas e assistência do professor, por meio da interação presencial. Ademais, a proposta alinha ensino-aprendizagem-avaliação com os resultados pretendidos da aprendizagem, conforme preconiza o Alinhamento Construtivo (Biggs; Tang, 2011).

\subsection{Aprendizagem Baseada em Projetos (ABP)}

A ABP pode ser entendida como uma abordagem pedagógica que tem sido utilizada em diversas instituições de ensino ao redor do mundo. $\mathrm{Na}$ ABP, os alunos analisam problemas contextualizados como mundo real e, de maneira individual ou colaborativa, decidem a forma mais significativa de abordá-los, em busca de soluções. Para que esse ensino seja mais interessante, procura-se promover a aplicação de problemas, tarefas e questões que sejam altamente motivadoras e significativas para os alunos (Bender, 2014). Os trabalhos em grupo são parte importante dessa aprendizagem. Nessas atividades, as tarefas são dividas, buscando explorar as melhores características de cada aluno.

Segundo Bender (2014), metodologicamente, a ABP parte de uma âncora, isto é, uma pequena descrição do problema a ser analisado. A partir disso, definem-se quais serão as questões motrizes, cujos objetivos irão delinear os parâmetros específicos do trabalho dos alunos. Assim, iniciam-se os processos científicos para investigação e pesquisa, onde atividades e processos, que não estavam previstos nas questões iniciais, surgem naturalmente dentro do projeto pela ação direta dos alunos. Nesse ponto, o professor passa a atuar predominantemente como um orientador. Ao final, os alunos recebem feedback de suas investigações e os resultados são apresentados publicamente.

Os projetos práticos funcionam como elementos catalisadores para a aprendizagem, uma vez que, por meio da solução de problemas contextualizados com a realidade dos alunos, incentiva-se o desenvolvimento do pensamento crítico e o engajamento dos mesmos na solução dos problemas propostos. Além disso, ABP incentiva o desenvolvimento intelectual autônomo dos alunos, ao mesmo tempo em que promove as relações coletivas e o espírito de grupo.

No contexto deste trabalho, a administração dos conteúdos e resolução dos projetos foi planejada considerando o modelo de Sala de Aula Invertida, conforme descrito na próxima seção.

\subsection{Sala de Aula Invertida}

Para a melhor compreensão do modelo de Sala de Aula Invertida, deve-se primeiramente entender o conceito de Ensino Híbrido. Atribui-se esse nome a qualquer programa educacional formal no qual o estudante aprende, pelo menos em parte, por meio do ensino on-line, com algum elemento de controle sobre o tempo, o lugar, o caminho e/ou ritmo (Christensen et al., 2013) e, em parte, pelo ensino presencial.

A forma de implementação do ensino híbrido pode seguir diferentes modelos, no caso deste trabalho, foi adotado o modelo de Sala de Aula Invertida, no qual parte do conteúdo é aprendido de forma autônoma pelo aluno, fora de sala de aula, com suporte de diferentes recursos (aulas em vídeo, textos, exemplos, entre outros) e o momento presencial, em sala de aula, é dedicado a supervisão e orientação do professor para tirar dúvidas, reforçar determinados conteúdos, auxiliar na resolução de problemas e dar feedback aos alunos. Os conteúdos on-line planejados e preparados pelo professor devem contribuir para a solução dos projetos desenvolvidos pelos alunos, preparando os estudantes para as atividades das aulas seguintes. A opção por esse modelo de ensino está pautada na sua capacidade de construção e aplicação fluida de planos individuais de aprendizado, bem como de incentivar o desenvolvimento autônomo dos alunos. 
Por fim, cabe destacar que a proposta de ensino-aprendizagem teve seu planejamento de ensino orientado pelo Alinhamento Construtivo de Biggs e Tang (2011) e essas orientações são apresentadas na seção seguinte.

\subsection{Alinhamento Construtivo}

Segundo o Alinhamento Construtivo (Biggs; Tang, 2011), o planejamento de ensino deve iniciar com a definição dos resultados pretendidos da aprendizagem (RPA), isto é, com a clara definição do que os alunos serão capazes de realizar após aprenderem determinado conteúdo ou passarem por uma determinada aula. Com base nos RPA, devem ser projetadas as atividades de ensino e aprendizagem (AEA) que capacitem os alunos a executar as ações previstas nesses RPA. Por fim, devem ser planejadas as tarefas de avaliação (TA), as quais devem ser capazes de mensurar quão bem os alunos atingiram os RPA. Portanto, AEA e TA devem estar alinhadas com os RPA.

No contexto deste trabalho, rubricas foram utilizadas para avaliar o desempenho dos estudantes e podem ser compreendidas como um conjunto de critérios sobre o trabalho a ser realizado pelos alunos que inclui descrições de níveis de desempenho (por exemplo, iniciante, próximo de proficiente, proficiente e avançado). Esses critérios devem ser claros e apropriados, a fim de permitir que os trabalhos realizados pelos alunos possam ser avaliados e classificados segundo os níveis de desempenho estabelecidos (Brookhart, 2013).

\section{Materiais e Métodos}

Nesta seção serão apresentados os detalhes da concepção da proposta de ensinoaprendizagem, incluindo a organização dos conteúdos, o planejamento das práticas, a dinâmica implementada, a seleção das tecnologias, o percurso metodológico para avaliação da proposta de ensino-aprendizagem e do produto educacional gerado, assim como os seus resultados da avaliação tanto da proposta de ensino-aprendizagem, quando do produto educacional.

\subsection{Resultados Pretendidos da Aprendizagem, Conteúdo e Carga Horária}

A proposta de ensino-aprendizagem foi planejada para favorecer o alcance dos seguintes RPA pelos alunos: (i) identificar as ferramentas básicas e as características das imagens de Radar por meio da descrição da ação realizada; (ii) processar uma imagem de Radar orbital; e (iii) interpretar uma imagem de Radar orbital para a realização de um diagnóstico ambiental.

A proposta foi implementada na forma de um curso, com uma carga horária de 30 (trinta) horas, sendo organizado em 04 (quatro) módulos. O primeiro módulo contém informações introdutórias sobre o funcionamento dos satélites imageadores e as características básicas das imagens de Radares orbitais, sua importância, aplicações e, principalmente, as diferenças entre elas e as imagens oriundas de sensores passivos. No segundo módulo, ocorre o aprofundamento dos conteúdos relacionados à obtenção e análise de imagens ópticas, além da utilização do software de geoprocessamento. No terceiro módulo, são iniciados os trabalhos com as imagens de Radar e, por esse motivo, são apresentados, além das características técnicas específicas dos sensores ativos, os procedimentos para obtenção, processamento e interpretação dessas imagens.

Nos primeiros 03 (três) módulos, os alunos resolvem, pequenos problemas como forma de fixar os conteúdos, lidar com as tecnologias e desenvolver, gradativamente, as habilidades para interpretação e análise de imagens de Radar. No quarto módulo, são resolvidos projetos que integrem os conteúdos ensinados nos módulos anteriores. Portanto, como pode ser observado, todo o curso é baseado em atividades práticas. V. $18 \mathrm{~N}^{\circ} 1$, julho, 2020 RENOTE DOI: 


\subsection{Projetos}

Os projetos e problemas utilizados eram condizentes com o futuro profissional dos alunos, envolvendo temas importantes e abrangentes, cujas técnicas de interpretação de imagem podem ser replicadas em outros tipos de análises. Por exemplo: com a mesma técnica de análise de desmatamento (extração vegetal), pode ser analisada uma queimada; da análise de uma área alagada pode-se analisar um represamento e assim por diante. Além disso, os atores dos problemas propostos representam diferentes carreiras profissionais que se utilizam das informações obtidas por meio de sensoriamento remoto, exemplo, fiscal do IBAMA, pesquisador do ICMBio, profissional da FUNAI, ANP, Defesa Civil, Polícia Federal, etc.

\subsection{Dinâmica de ensino}

Os conteúdos e as atividades didáticas foram ministrados segundo um gradiente de complexidade, de acordo com a evolução dos módulos, conforme ilustrado na Figura 1 e os conteúdos on-line foram administrados por meio da plataforma Google Classroom, mesclando conteúdo e pequenas tarefas que estimulavam a aprendizagem autônoma dos alunos, tanto dos aspectos teóricos, quanto da prática com o uso das tecnologias.

\begin{tabular}{|c|c|c|c|c|}
\hline CONTEÚDOS & $\begin{array}{l}\text { INTRODUÇÃO AO } \\
\text { SENSORIAMENTO } \\
\text { REMOTO }\end{array}$ & $\begin{array}{c}\text { OBTENÇÃO E } \\
\text { ANÁLISE DE } \\
\text { IMAGENS ÓPTICAS }\end{array}$ & $\begin{array}{c}\text { OBTENÇÃO E } \\
\text { ANÁLISE DE } \\
\text { IMAGENS DE RADAR } \\
\text { SENTINEL-1 }\end{array}$ & $\begin{array}{l}\text { SOLUÇÃO DE } \\
\text { PROBLEMAS }\end{array}$ \\
\hline \multirow[t]{2}{*}{ ATIVIDADES } & $\begin{array}{c}\text { PRÁTICAS } \\
\text { DIRIGIDAS EM SALA } \\
\text { DE AULA } \\
+ \\
\text { PRÁTICAS } \\
\text { DIRIGIDAS } O N-L I N E\end{array}$ & $\begin{array}{c}\text { PRÁTICAS } \\
\text { DIRIGIDAS EM SALA } \\
\text { DE AULA } \\
+ \\
\text { PRÁTICAS } \\
\text { DIRIGIDAS ON-LINE }\end{array}$ & $\begin{array}{c}\text { PRÁTICAS } \\
\text { DIRIGIDAS EM SALA } \\
\text { DE AULA } \\
+ \\
\text { PRÁTICAS } \\
\text { DIRIGIDAS ON-LINE }\end{array}$ & $\begin{array}{c}\text { PRÁTICAS } \\
\text { AUTÔNOMAS } \\
\text { INDIVIDUAIS E EM } \\
\text { GRUPO } \\
+ \\
\text { PRÁTICAS } \\
\text { DIRIGIDAS ON-LINE }\end{array}$ \\
\hline & MÓDULO I & MÓDULO II & MÓDULO III & MÓDULO IV \\
\hline
\end{tabular}

Figura 1 - Gradiente de evolução dos conteúdos e atividades didáticas (Autoria própria).

Na avaliação, foi observado o alcance dos 03 (três) RPA descritos na Seção 3.1. Para cada RPA, foram definidos quatro níveis de desempenho (avançado, proficiente, próximo de proficiente e iniciante), conforme ilustrado no Quadro 1.

Quadro 1 - Versão parcial da rubrica utilizada na avaliação.

\begin{tabular}{|c|c|c|c|c|}
\hline Con & do & Proficiente & Próx. Proficiente & Inic \\
\hline $\begin{array}{l}\text { Habilidade: } \\
\text { IDENTIFICAR } \\
\text { Ação: } \\
\text { Identificar as } \\
\text { ferramentas } \\
\text { básicas e as } \\
\text { características das } \\
\text { imagens de Radar } \\
\text { por meio da } \\
\text { descrição da ação } \\
\text { realizada. }\end{array}$ & $\begin{array}{l}\text { Identifica e } \\
\text { justifica de forma } \\
\text { correta, clara e } \\
\text { completa cada } \\
\text { uma das ações e } \\
\text { ferramentas } \\
\text { utilizadas para } \\
\text { solução da } \\
\text { situação- } \\
\text { problema. }\end{array}$ & $\begin{array}{l}\text { Identifica e justifica } \\
\text { corretamente cada } \\
\text { uma das ações e } \\
\text { ferramentas utilizadas } \\
\text { para solução da } \\
\text { situação-problema, } \\
\text { porém, sem o } \\
\text { detalhamento do nível } \\
\text { avançado ou por meio } \\
\text { da cópia do texto do } \\
\text { material de apoio. }\end{array}$ & $\begin{array}{c}\text { Identifica e/ou } \\
\text { justifica as ações e } \\
\text { ferramentas } \\
\text { utilizadas para a } \\
\text { solução da situação- } \\
\text { problema de forma } \\
\text { imprecisa, } \\
\text { diminuindo a clareza } \\
\text { e a completude da } \\
\text { resposta. }\end{array}$ & $\begin{array}{l}\text { Identifica e/ou } \\
\text { justifica as } \\
\text { ações e } \\
\text { ferramentas } \\
\text { utilizadas para } \\
\text { a solução da } \\
\text { situação- } \\
\text { problema de } \\
\text { forma } \\
\text { incoerente ou } \\
\text { incorreta. }\end{array}$ \\
\hline
\end{tabular}

Fonte: Autoria própria.

\subsection{Tecnologias}

Considerando as opções de geotecnologias e repositórios disponíveis atualmente e o contexto educacional brasileiro, optou-se pela escolha exclusiva de recursos gratuitos, 
multiplataforma e adequados para aplicações profissionais e acadêmicas, conforme listados no Quadro 2.

Quadro 2 - Geotecnologias e repositórios utilizados no estudo de caso.

\begin{tabular}{|l|l|l|}
\hline \multicolumn{1}{|c|}{ Recursos } & \multicolumn{1}{|c|}{ Descrição } & \multicolumn{1}{c|}{ Fonte } \\
\hline QGIS & Sistema de informação geográfica & www.qgis.org \\
\hline SNAP & Sistema de processamento digital de imagens & step.esa.int/main/download \\
\hline Serviço Geológico dos EUA & Repositório das imagens Landsat-8 & earthexplorer.usgs.gov \\
\hline Agência Espacial Européia & Repositório das imagens da missão Sentinel & scihub.copernicus.eu/dhus \\
\hline
\end{tabular}

\section{Fonte: Autoria própria.}

\subsection{Avaliação da proposta de ensino-aprendizagem}

A eficiência da proposta de ensino-aprendizagem foi avaliada por meio de uma aplicação prática, realizada no período de 06 a 10 de agosto de 2018, em uma instituição federal de ensino superior e implementada no formato de um curso de extensão denominado 'Diagnósticos ambientais por meio de imagens de Radar', cujas inscrições ocorreram de maneira voluntária e gratuita, por meio de um formulário eletrônico.

Esse estudo de caso contou com a participação de 12 (doze) sujeitos, sendo 06 (seis) homens e 06 (seis) mulheres, entre graduandos e recém-graduados dos cursos superiores de Engenharia Ambiental, Ciências Biológicas e Arquitetura, constituindo um grupo com faixa etária entre 24 (vinte e quatro) e 42 (quarenta e dois) anos de idade. Nesse universo, 50\% nunca havia realizado uma análise ambiental, $71 \%$ nunca havia realizado uma atividade prática de sensoriamento remoto e $79 \%$ nunca havia tido contato com uma imagem de Radar Sentinel-1. Essa heterogeneidade foi benéfica por colaborar para a avaliação, além da eficiência da proposta de ensino-aprendizagem, da sua transversalidade.

Para a avaliação das habilidades mencionadas na Seção 3.1, solicitamos aos alunos a resolução individual e autônoma do problema apresentado no Quadro 3, sendo que, para essa atividade, que funcionou como uma prova de conhecimento, os alunos receberam apenas 02 (duas) imagens de Radar Sentinel-1 da região de Manaus/AM, sendo uma de Maio/2017 e outra de Outubro/2018.

\section{Quadro 3 - Problema para avaliação do conhecimento.}

\section{Contextualização (situação hipotética):}

O alerta de cheia divulgado por um órgão de monitoramento geológico no final de 2017 informa que, em 2018, o Rio Negro não atingirá a cheia histórica de 2012. Todavia, com base nos índices pluviométricos previstos para essa região, a enchente do Rio Negro deve atingir cotas máximas equivalentes às registradas em Maio/2017. Com base no boletim hidrológico divulgado e a fim de planejar eventuais medidas de prevenção a desastres naturais, um grupo de Agentes da Defesa Civil foi acionado para avaliar quais seriam as comunidades possivelmente afetadas pela enchente de 2018.A você, como integrante desse grupo de estudo, coube a missão de avaliar se a comunidade ribeirinha do Bairro Educandos (Localização: Lat $3^{\circ} 08^{\prime} 22^{\prime \prime}$ S / Long 60 $00^{\prime} 43^{\prime \prime} \mathrm{W}$ ) corre o risco de ser atingida pela cheia do Rio Negro. Durante a divulgação do alerta de cheia, foram fornecidas imagens atualizadas da região de Manaus, bem como as imagens registradas na cheia de Maio/2017.

\section{Questões do Relatório:}

1 - Com base nas informações recebidas e nas imagens analisadas, pode-se concluir que a comunidade ribeirinha do Bairro Educandos (Localização: Lat 3 $08^{\prime} 22^{\prime \prime} \mathrm{S} /$ Long 60 $00^{\circ} 43^{\prime \prime} \mathrm{W}$ ) será atingida pela enchente do Rio Negro e, por esse motivo, medidas de proteção àquela comunidade devem ser tomadas? Como você chegou a essa conclusão?

2 - Descreva detalhadamente quais foram as técnicas e os recursos utilizados para chegar a essa conclusão, justificando o emprego de cada um deles.

Fonte: Autoria própria.

DOI: 


\subsection{Resultados da avaliação da proposta de ensino-aprendizagem}

Para o propósito deste artigo, os resultados serão apresentados com relação à avaliação da proposta de ensino-aprendizagem no que diz respeito ao alcance, pelos alunos, dos RPA descritos na Seção 3.1. Para isso, foi avaliado com o uso da rubrica (Quadro 1), o desempenho dos alunos a partir da resolução do problema apresentado no Quadro 3.

Nesse contexto, os resultados trazem evidências da efetividade da proposta de ensino-aprendizagem para o desenvolvimento das habilidades anteriormente mencionadas. Com base na rubrica elaborada para avaliação (ilustrada no Quadro 1), verificou-se que 33,3\% dos alunos alcançaram o nível 'Avançado' de desempenho, $58,3 \%$ o nível 'Proficiente' e apenas 8,4\% o nível 'Próximo de Proficiente'.

Isso significa dizer que, de forma autônoma e individual, os alunos demonstraram conseguir 'Interpretar' corretamente uma imagem de Radar e extrair dela as informações necessárias para o preenchimento de um relatório. Esse nível de desenvolvimento exige que os alunos saibam 'Processar' uma imagem de Radar Sentinel-1, além de 'Identificar' as características dessa imagem e as ferramentas utilizadas durante o processo.

Detalhando esses resultados, verificou-se que a habilidade 'Identificar' foi a que apresentou um nível de desenvolvimento médio ligeiramente inferior às demais. Por meio da observação presencial e da análise das respostas apresentadas pelos alunos, constatou-se que, embora se tratando da habilidade mais elementar a ser avaliada, foi tomada como menos importante pelos alunos, pois, aparentemente, eles não entenderam que seriam avaliados pela completude e detalhamento das descrições contidas naquelas respostas. Por esse motivo, mesmo possuindo a maior parte dos resultados dentro dos níveis considerados satisfatórios, foi a habilidade que apresentou maior número de resultados com o nível de desempenho Próximo ao Proficiente.

Com relação à habilidade 'Processar', considerada a mais complexa do ponto de vista procedimental, uma vez que contempla os processos específicos para tratamento de uma imagem de Radar Sentinel-1, observou-se que a proposta de ensinoaprendizagem foi eficiente para a obtenção de níveis de entendimento desejados, uma vez que, mesmo identificando alguns poucos resultados no nível de desempenho Próximo ao Proficiente, a maioria dos alunos atingiu o nível Proficiente ou Avançado.

Por fim, analisando os desempenhos dos alunos para a habilidade 'Interpretar', considerada a mais complexa do ponto de vista da abstração, uma vez que se refere à extração de informações de uma imagem de Radar Sentinel-1, constatou-se que a proposta de ensino-aprendizagem foi eficiente, pois todos os alunos atingiram o nível de desempenho Proficiente ou Avançado.

\subsection{Concepção e avaliação do Produto Educacional}

Considerando os resultados positivos obtidos com relação à avaliação da proposta de ensino-aprendizagem, esse conteúdo didático foi sistematizado em um 'Curso de Introdução ao Processamento e à Interpretação de Imagens Sentinel-1', o qual tem como objetivo auxiliar professores de geotecnologias a introduzirem, de forma prática, essa temática para suas turmas de alunos e que, respeitados os direitos autorais, pode ser acessado gratuitamente no endereço: http://ppget.ifam.edu.br/dissertacoes-defendidas/.

Esse curso, foi formatado como um 'Guia Didático para Professores', além de possuir uma identidade visual própria, está organizado em 04 (quatro) módulos e, em cada um deles, o professor recebe orientações sobre como selecionar o conteúdo teórico e organizar a sua sala de aula, sendo também orientado sobre quais são os objetivos pedagógicos de cada etapa do curso e os recursos tecnológicos mínimos necessários para a sua execução. Em todas as etapas, o professor tem acesso a exemplos e sugestões V. $18 \mathrm{~N}^{\circ} 1$, julho, 2020 RENOTE DOI: 
de atividades práticas que o auxiliarão no ensino de assuntos relacionados ao processamento e a interpretação de imagens de Radar Sentinel-1.

Nesse contexto, buscando avaliar a escalabilidade desse produto educacional, bem como a sua capacidade de ser replicado em diferentes contextos educacionais, o 'Curso de Introdução ao Processamento e à Interpretação de Imagens Sentinel-1 - Guia Didático para Professores' foi avaliado por professores, mestres e doutores que lecionam formações relacionadas com sensoriamento remoto e geotecnologias.

Inicialmente, foi selecionado e convidado um comitê ad hoc tão diversificado como são diversificados os níveis de formação e as especialidades dos professores que ministram disciplinas relacionadas a geotecnologias. Esse grupo de avaliadores foi composto por 03 (três) homens e 01 (uma) mulher, entre 20 (vinte) a 50 (cinquenta) anos de idade, oriundos dos cursos de Geografia, Engenharia Civil e Gestão Ambiental, sendo 01 (um) Doutor, 01 (um) Mestre, 01 (um) Graduado em Licenciatura e 01 (um) Tecnólogo.

Em seguida, todos os professores-avaliadores receberam, em formato digital (.pdf), uma cópia do "Guia Didático para Professores" e foram orientados, individualmente, a proceder da seguinte forma: (i) o professor deveria analisar o produto educacional que recebeu, avaliando-o segundo sua perspectiva de atuação e o seu contexto educacional; (ii) em seguida, com base na sua análise, o professor deveria preencher o questionário de avaliação do produto educacional que continha 17 (dezessete) questões fechadas sobre a qualidade, a organização, a relevância, a adequação e a replicabilidade do curso proposto, além de 03 (três) questões abertas nas quais os avaliadores poderiam opinar sobre eventuais dificuldades para replicação, pontos fortes e oportunidades de melhoria.

Os resultados obtidos das avaliações realizadas pelos professores, mestres e doutores trazem evidências de que o produto educacional, além de adequado e relevante, é replicável em diferentes contextos educacionais.

Da análise dos dados obtidos, observou-se que os avaliadores foram unânimes em afirmar que: (i) sentem-se capazes de reproduzir o curso, segundo as orientações contidas no "Guia do Professor"; (ii) acreditam que as orientações contidas no "Guia do Professor" são replicáveis em diferentes contextos educacionais; (iii) avaliam que as orientações conseguem atingir os objetivos propostos, ou seja, orientar o professor na realização de um "Curso de Introdução ao Processamento e à Interpretação de Imagens Sentinel-1; (iv) acreditam que, no caso da replicação, os alunos teriam condições de adquirir noções básicas de processamento e interpretação das imagens Sentinel-1.

\section{Considerações Finais}

Neste artigo apresentou-se uma proposta de ensino-aprendizagem e um produto educacional direcionado para professores de geotecnologias em Curso Superiores ligados a área de Meio Ambiente, a fim de favorecer o ensino de interpretação e análise de imagens de Radar Sentinel-1.

Do ponto de vista do ensino, acredita-se que essa pesquisa contribui significativamente para ampliar as perspectivas de formação de estudantes de cursos superiores da área de Meio Ambiente, na medida em que traz luz para os conteúdos referentes aos sensores ativos, os quais são pouco evidenciados em cursos de graduação. Além disso, apresenta uma dinâmica de aula fundamentada no emprego de softwares livre e em metodologias ativas, que incentivam a aprendizagem autônoma por parte dos alunos, a resolução de problemas práticos e a organização dos recursos de estudo com vistas a promover aprendizagem dentro e fora do espaço de sala de aula. 
Como trabalhos futuros, planeja-se ampliar os resultados da pesquisa, com base em replicações do estudo e também elaborar um material didático para ser utilizado diretamente por estudantes e, ainda, a formatação deste produto educacional para ser ministrado em uma plataforma de ensino a distância (EAD).

\section{Referências}

AGÊNCIA ESPACIAL BRASILEIRA. Nasa Globe, 2019. Disponível em: <http://www.aeb.gov.br/espaco-educacao-e-tecnologia/nasa-globe/>. Acesso em: 28 de abril de 2019.

BENDER, W. N. Aprendizagem baseada em projetos - Educação diferenciada para o Século XXI. Porto Alegre: Penso, 2014.

BIGGS, J.; TANG, C. Teaching for Quality Learning at University. 4. ed. Berkshire, England: Society for Researching to Higher Education \& Open University Press, 2011.

BROOKHART, S. M. How to create and use rubrics for formative assessment and grading. Alexandria, VA: ASCD, 2013.

CHRISTENSEN, C.; HORN, M.; STAKER, H. Ensino Híbrido: uma Inovação Disruptiva? Uma introdução à teoria dos híbridos. Clayton Christensen Institute for disruptive innovation, 2013. Disponível em: <https://porvir.org/wpcontent/uploads/2014/08/PT_Is-K-12-blended-learning-disruptive-Final.pdf > .Acesso em: 06 de julho de 2020.

DIAS, J. C.; MENDONÇA, A. P. Diagnósticos ambientais por meio da interpretação de imagens de Radares orbitais: algumas questões sobre o ensino. In: Coelho, I. M. W. da S. ...[et al.](Org.). In: Perspectivas metodológicas para sala de aula. $1^{\mathrm{a}}$ ed. Curitiba: Appris, 2019.

EMBRAPA - Atlas escolar da região metropolitana de Campinas, 2020. Disponível em: <https://www.embrapa.br/busca-de-publicacoes/-/publicacao/988128/atlas-escolarda-regiao-metropolitana-de-campinas>. Acesso em: 12 de maio de 2020.

GEODEN - Geotecnologias Digitais no Ensino, 2019. Disponível em: <http://www.geoden.uff.br/>. Acesso em: 28 de abril de 2019.

INSTITUTO BRASILEIRO DE GEOGRAFIA E ESTATÍSTICA - Um modelo metodológico de classificação de climas. Brasília, 2002. Disponível em:

<http://geoftp.ibge.gov.br/produtos_educacionais/atlas_educacionais/atlas_geografico_e scolar/mapas_do_brasil/mapas_nacionais/informacoes_ambientais/brasil_clima.pdf $>$. Acesso em: 22 de outubro de 2018

JENSEN, J. R. Sensoriamento Remoto do Ambiente: Uma Perspectiva em Recursos Terrestres. Tradução José Carlos Neves Epiphanio (coordenador)...[et al.]. 2. ed. São José dos Campos, SP: Parêntese, 2009.

NOVO, E. M. L. de M. Sensoriamento remoto: princípios e aplicações. São Paulo: Ed. Blucher, 2010. 363p.

SIMON, A. L. H.; CUNHA, C. M. L. Utilização de imagens do Google Earth na identificação de feições geomorfológicas antropogênicas. In: Simpósio de Pós-

Graduação em Geografia do Estado de São Paulo. Anais. Rio Claro-SP, 2008.

SOUZA, R. B.Sensoriamento Remoto: conceitos fundamentais e plataformas. In: IV CEOS WGEdu Workshop. Anais. Santa Maria-RS: INPE, 2010.

V. $18 \mathrm{~N}^{\mathrm{o}} 1$, julho, 2020 RENOTE DOI: 